\title{
MECHANISMS FOR DYNAMO MODE EXCITATION
}

\author{
P. HOYNG \\ Laboratory for Space Research \\ Sorbonnelaan 2, 3584 CA Utrecht \\ The Netherlands
}

\section{(extended summary)}

Dynamo theory of the solar magnetic field finds itself in a period of reappraisal since about 1980 . There are two reasons for this: (1) The required increase of the angular speed $\Omega$ with decreasing $r$ is neither found in numerical simulations nor measured by helioseismology. (2) Mean field modeling shows often little regard for inherent physical restrictions and selfconsistency.

In recent years, attention has shifted from the convection zone to the boundary layer between convection zone and radiative interior as a more likely location for the solar dynamo (Rai Choudhuri 1990a,b). The topic of dynamo mode excitation, however, is model-independent and starts from the observation that kinematic $\alpha \Omega$ dynamos are strictly periodic in time. Only the fundamental mode is excited and it has an infinite phase memory, which is not satisfactory.

The reader is referred to the proceedings of the Kiev symposium for the complete text of my review (Hoyng 1990). Here I shall restrict myself to an extended summary, giving only new references that came to my attention during the last half year.

\section{Mode Excitation in Mean Field Theory}

The transport properties of the mean field $\langle\mathrm{B}>$ are determined by the dynamo equation. Its basic form is:

$$
\partial_{t}<\mathbf{B}>=\mathbf{D} \cdot\left\langle\mathbf{B}>+\mathbf{F} ; \quad \mathbf{D}=\nabla \times\left(\mathbf{v}_{0} \times+\alpha-\beta \nabla \times\right) .\right.
$$

Here, $\mathbf{v}_{\mathbf{0}}$ is the mean flow, and $\alpha$ and $\beta$ are the usual dynamo coefficients related to the turbulent velocity $\mathbf{v}$ superposed on $\mathbf{v}_{0}$. Tradition has it that $\langle\mathbf{B}\rangle$ is a spatial average over an unspecified scale in between the radius $R$ of the dynamo and the eddy size $\lambda_{c}$. The term $\mathbf{F}$ collects all terms due to the fact that, among other things, $\langle f\langle g \gg \neq\langle f\rangle\langle g\rangle$. F is always neglected on the ground that it is formally of 
order $\lambda_{c} / R \ll 1$. The growth rate of an eigenmode of eq. (1.1) is determined by the dynamo number ( $\Delta \Omega$ is the difference in angular velocity in the dynamo):

$$
D=\alpha \Delta \Omega R^{3} / 2 \beta^{2} ; \quad D_{c} \simeq(2 \pi)^{3} .
$$

(a). Instability $\left(D \geq D_{c}\right)$. One or more eigenmodes will grow until nonlinear effects become important. A popular idea has been that these reduce $D$ to $D_{c}$, e.g. by reducing $\alpha$. Only the fundamental mode is then excited. The dynamo has a single frequency $\omega$ and an infinite quality factor $Q=\omega / \Delta \omega$. The fine tuning of $D$ may not be stable, leading to complicated nonlinear interactions between magnetic and fluid modes. In that case the dynamo has several frequencies, each with a finite $Q$. (b). External forcing $\left(D<D_{c}\right)$. All eigenmodes are damped so that $\langle\mathbf{B}>\rightarrow 0$. Subcritical mode excitation may occur if $\mathbf{F}$ in eq. (1.1) is sufficiently large. $\mathbf{F}$ depends on $\mathbf{v}$ and has the character of a random forcing term. This would lead to excitation of many eigenmodes, each with a finite $Q$.

Another external forcing mechanism could be a relic field in the radiative core, which imposes a nonzero boundary condition on eq. (1.1) at the base of the convection zone. This boundary condition could even be periodic, in which case the phase stability of the dynamo at that frequency may be very high.

\section{Observations}

Variations in the 22-yr period. From the epochs of sunspot extrema one finds $\delta P_{r m s} / P \sim 0.1$, where $P=$ mean half cycle period. It is not known if subsequent period variations $\delta P$ are independent or whether the cycle is rather a passive and noisy reflection of a high $Q$ oscillator in the solar core. There are marginal indications for a random walk in phase (which would exclude a core oscillation), but the data set ( $A D 1610$ - present) is really too small to draw a conclusion. The phase stability of the 22-yr cycle may ultimately be determined from the ${ }^{14} \mathrm{C}$ data and of the ${ }^{10} \mathrm{Be}$ record discovered in Greenlandic ice cores.

Longer periods. Several proxy records indicate irregular modulations in the level of the activity cycle with a time scale between one and a few hundred years. From an analysis of the 88-yr cycle Feynman and Gabriel (1990) have argued that the solar dynamo is operating in a region close to the transition between period doubling and chaos. Attempts have been made to determine attractor dimensions from the sunspot and ${ }^{14} \mathrm{C}$ records but the problem is again the length and quality of the data set. It is recalled that Barnes et al. obtained a remarkable similarity with the observed solar cycle, including Maunder-type minima, by simulating yearly sunspot numbers from narrowband Gaussian noise.

Periods shorter than 22 year. Stenflo and coworkers have analysed 25 years of synoptic magnetograph data and discovered that the surface magnetic field exhibits well-defined periods down to 1.5 year. This has been interpreted as excitation of overtones of the dynamo equation. Gokhale and Javaraiah (1990) have extended 
the dataset by using magnetic field data which they simulated from the Greenwich sunspot data (but they have not yet analysed the power diagram at even $\ell$ ).

\section{Nonlinear mode excitation}

I shall restrict myself to nonlinear mean field models. Three types of nonlinearity have been considered since the very beginning: quenching of the $\alpha$-effect, flux losses through buoyancy, and suppression of the differential rotation. Early nonlinear studies established the existence of stable nonlinear dynamo waves, but did not systematically explore the behaviour of the dynamo as a function of position in parameter space. That was only done after about 1980, when mean field modeling fell in love with nonlinear dynamics. Initial studies in this area truncated all spatial dependence by replacing $\nabla$ by $\ell^{-1}$. In return for extreme simplification they discovered that the solar dynamo might have chaotic properties. Ruzmaikin has suggested that the 'grand minima' in the solar cycle may correspond to a strange attractor, entirely in line with the fashion of that time. It took many years before more realistic attempts were undertaken in which the spatial structure is taken into account. Three such models have recently been published (Schmitt and Schüssler 1989, Brandenburg et al. 1990, and Belvedere et al. 1990).

Nonlinear mean field theory is still in a very early stage of development. On the one hand, very interesting dynamical behaviour is discovered, sometimes strikingly similar to what is observed, but on the other hand the solutions are often very sensitive to the adopted functional form of the nonlinearity and/or the values of parameters. To make things worse, the nonlinearities are always introduced phenomenologically. It is therefore not yet possible, for example, to predict the magnitude of $\langle\mathbf{B}>$. Dynamical selfconsistency is the outstanding problem in nonlinear mean field theory, and in my view the prospects for progress are not very bright.

\section{Mode excitation by external forcing}

External forcing may be effectuated through boundary conditions or through fluctuations in the turbulent convection. A fossil field in the core would impose a boundary condition $\langle\mathbf{B}\rangle=\mathbf{B}_{\text {core }}$ at the base of the convection zone. The field will be smeared out by the turbulent dynamo, but there remains a net polarity and intensity asymmetry in the activity cycle. Models have been constructed along these lines, and the conclusion is that $\mathbf{B}_{\text {core }} \lesssim 1 \mathrm{G}$ at the bottom of the convection zone. It has also been suggested that the solar core performs a 22 -yr torsional oscillation with the poloidal component of the field acting as a spring. The dynamo in the convection zone would then be in a state of forced oscillation. This remains an interesting idea, although a credible model has never been elaborated.

External forcing by fluctuations in the turbulence (the term $\mathbf{F}$ in (1.1)) has received little attention. One possibility is that a sudden burst in the cyclonic 
convection causes a jump in $\alpha$, and it has been shown that this may induce a reversal of the (mean) geomagnetic field in linear theory. The conventional wisdom of today is that things like reversals in $\alpha^{2}$ dynamos and aperiodicity in $\alpha \Omega$ dynamos are caused by nonlinearities. However, even in linear theory the periods of adjacent cycles of an $\alpha \Omega$ dynamo must differ, as the realisation of the turbulence is different. It should be possible to formulate linear mean field theory in the same way as a scalar diffusion process, with a continuous loss of memory.

This is again the effect of the random forcing term F, but it is extremely difficult to evaluate its magnitude. This problem has recently been tackled by using ensemble rather than spatial averaging. It has been shown that the large scale field of an $\alpha \Omega$ dynamo behaves as a quasiperiodic random function. The period stability of the solar dynamo is currently being evaluated. A rough estimate gives $\delta P_{r m s} / P \sim 1$, highlighting the potentially enormous influence of $\mathbf{F}$ in the linear theory.

\section{References}

Belvedere, G., Pidatella, R.M. and Proctor, R.M.E.: 1989, Geophys. Astrophys. Fluid Dynamics, to appear.

Brandenburg, A., Moss, D. and Tuominen, I.: 1989, Geophys. Astrophys. Fluid Dynamics, to appear.

Feynman J. and Gabriel, S.B.: 1990, Solar Physics, to appear.

Gokhale, M.H. and Javaraiah, J.: 1990, M.N.R.A.S., to appear.

Hoyng, P.: 1990, in Solar photosphere: Structure, Convection, and Magnetic fields, ed. J.O. Stenflo, Kluwer (Dordrecht), p. 359.

Rai Choudhuri, A.: 1990a, these proceedings.

Rai Choudhuri, A.: 1990b, Astrophys. J., to appear.

Schmitt, D. and Schüssler, M.: 1989, Astron. Astrophys. 223, 343.

\section{DISCUSSION}

VAN BALLEGOOIJEN: What are the prospects for direct numerical 3D simulations, without invoking the mean-field approximation?

HOYNG: These 3D simulations fail at present to reproduce an acceptable solar cycle. One of the reasons is suspected to be inadequate spatial resolution. The contribution of subgrid processes must always be parametrised (one might say that a kind of mean field approximation is implicit, albeit on a rather small spatial scale). It is my understanding that there is no hope of being able to resolve eddies of the required size in the foreseeable future in solar dynamo models.

WEISS: Modern supercomputers are powerful enough to model selfconsistent nonlinear dynamos in idealized geometries with parametrized diffusion coefficients. However, it seems premature to embark on such a detailed computation until we understand more about the location of the dynamo and the variation of angular velocity near the base of the convection zone. 
HOLLWEG: Is the 153-day periodicity of solar flares a proxy indicator of yet another fundamental dynamo period?

HOYNG: It could be.

SILVARAM: Would you clarify the role of ${ }^{10} \mathrm{Be}$ in detecting variations in activity? What range of periods does it cover and how does it compare with ${ }^{14} \mathrm{C}$ ?

HOYNG: ${ }^{10} \mathrm{Be}$ and ${ }^{14} \mathrm{C}$ are produced by (solar) cosmic rays interacting with the Earth's atmosphere. ${ }^{10} \mathrm{Be}$ gets attached to aerosols and is removed by precipitation after a typical time of 1 or 2 years (much faster than ${ }^{14} \mathrm{C}$ ). Because of its half-life time of $1.5 \times 10^{6}$ year (as compared to $5730 \mathrm{yr}$ for ${ }^{14} \mathrm{C}$ ) the ${ }^{10} \mathrm{Be}$ proxy record in ice cores reaches much farther back in time, at least in principle. Variations in ${ }^{14} \mathrm{C}$ and ${ }^{10} \mathrm{Be}$ records over the last $10^{4}$ year show a very encouraging correlation.

AL-KHASHLAN: If the number of relevant dynamical variables is very low what is the behaviour of the dynamo?

HOYNG: Models for the solar dynamo with only a few dynamical variables have been published for example by Ruzmaikin (Comm. Astrophys. 9 (1981)85), Krause and Roberts (Adv. Space Res. 1 (1981)231) and by Weiss, Cattaneo and Jones (Geophys. Astrophys. Fluid Dyn. 30 (1984)305). The nature of dynamo action is field amplification by shear flows (of the right kind), to balance loss processes such as ohmic decay, losses through buoyancy, etc. 